\title{
Reduction of uncertainties in exposure assessment based on environmental monitoring data
}

\author{
M. Bleher ${ }^{1, *}$, F. Gering ${ }^{1}$, U. Stöhlker ${ }^{1}$, T. Karhunen ${ }^{2}$, A. Nalbandyan-Schwarz ${ }^{3}$, \\ C. Woda ${ }^{4}$ and A. Mafodda ${ }^{4}$ \\ ${ }^{1}$ BfS - Bundesamt für Strahlenschutz, Neuherberg and Freiburg, Germany. \\ 2 STUK - Radiation and Nuclear Safety Authority, Helsinki, Finland. \\ ${ }^{3}$ DSA - Norwegian Radiation and Nuclear Safety Authority, Tromsø, Norway. \\ ${ }^{4}$ HMGU - Helmholtz Zentrum München-German Research Center for Environmental Health, Neuherberg, Germany.
}

\begin{abstract}
Emergency preparedness and response systems for nuclear and radiological emergencies have to deal with decision-making in situations with relevant uncertainties. Consistent and appropriate protective measures must be decided before, during and after emergency situations. CONFIDENCE WP2 research helps to improve this decision-making process in the urgent response and the early response phase of emergency situations with potential major releases to atmosphere. This paper describes methods to reduce uncertainties in dose assessment for the population using data from stationary and mobile environmental monitoring programs. A special focus is given to identification of the measurement uncertainties of stationary and mobile monitoring systems. Methods to reduce these uncertainties and procedures to optimise mobile monitoring strategies are discussed. A first contribution towards assessing the quality of dose-rate measurements performed by the general population is made. In addition, the paper introduces approaches for advanced dose assessment tools using monitoring data and concepts for identifying critically exposed groups.
\end{abstract}

Keywords: emergency response / monitoring strategies / dose assessment / reduction of uncertainties / citizen engagement in dosimetry

\section{Introduction}

Emergency preparedness and response systems for nuclear and radiological emergencies have to deal with decisionmaking in situations with relevant uncertainties. Consistent and appropriate protective measures must be decided before, during and after such emergency situations. This requires information about the affected areas, the level of contamination and the actual and future exposure.

Nuclear power plant accidents in Chernobyl (1986) and in Fukushima Daiichi (2011) prove that environmental monitoring data are very important for assessing the exposure to population in affected areas. Both accidents were characterized by released radioactive materials with activities above $100 \mathrm{PBq}$ and the duration of the release exceed one week. In both cases, data from stationary monitoring systems provided only a little information about the situation. Extended mobile monitoring programs were needed to provide detailed

\footnotetext{
* Corresponding author: mbleher@bfs.de
}

information on the exposure situation. To improve the monitoring capacity, national dose rate monitoring systems were improved after the Chernobyl accident in many countries. In addition, the European data exchange platform EURDEP was introduced to trigger the exchange of information in emergency situations with transboundary aspects.

In some cases, a pre-release phase may occur - like in the Fukushima Daiichi accident, where a major release of radioactive material into the atmosphere occurred nearly one day after the initiating event. Decision support systems allow an assessment of the impact of a potential release especially in such a pre-release phase. Furthermore, such systems allow an assessment of the potential exposure to the public and time to propose adequate protective actions. The most relevant protective actions in this phase are evacuation, preparing sheltering and distribution of stable iodine tables.

The release phase of an event starts, when significant amounts of radioactive material are released to the atmosphere (or to water systems like rivers). The release phase ends, when the release has stopped, and the risk of additional further releases is small. The most relevant protective actions in this 
phase are sheltering and administration of stable iodine tables. In addition, consumption of potential contaminated local produced agricultural products and drinking water should be avoided.

IAEA GSG-11 report (IAEA, 2018) denotes pre-release and release phase together as the urgent response phase.

The post-release phase or early response phase starts, once the contaminated cloud has passed the region of interest. The most relevant protective actions in this phase are sheltering (for a short period of time) and/or relocation of people living in area with an assessed exposure exceeding a given intervention level. In addition, the consumption of contaminated local produced agricultural products and drinking water should be avoided. If there is no need for further emergency response actions, the post-release phase can be terminated and a "transition phase" may start (c.f. WP4).

Most countries introduced emergency preparedness and response systems based on different assumptions about types of release scenarios as a planning basis. National threat analysis investigations depend on historical events as well as on safety analysis for existing facilities. To compare, in the Nordic countries - Denmark, Finland, Sweden, Norway and Iceland -, the scope of definitions is broader and in places more specific than those existing in, for example, the IAEA guides (Bleher et al., 2019).

WP2 of the European project CONFIDENCE deals with the reduction of uncertainty in dose assessment for the population. This research aims to improve the preparedness and response capabilities in the early phase of a major accidental release situation. It addresses different aspects of potential releases to atmosphere and discusses methods to improve environmental monitoring strategies including stationary monitoring and monitoring by mobile teams. Concepts for identifying critically exposed groups are also introduced and discussed.

\section{Objectives}

In the very early (pre-release) phase of accidental release scenarios, decision support systems like JRODOS and ARGOS (see e.g. Ehrhardt and Weis, 2000; Raskob and Gering, 2010; Ievdin et al., 2010; Gering et al., 2010) have to rely on prognostic model calculations. Figure 1 gives an overview of the JRODOS model chain and related uncertainty budgets. The impact of a major release scenario mainly depends on the total amount and the nuclide composition of the release. The source term describes the amount of the released material and start, duration and time dependence of released activity concentration for relevant nuclides.

JRODOS and ARGOS use output from dispersion models as a basis for dose assessment. Inhalation dose and external dose from the cloud shine can be assessed from (time integrated) air activity concentration and assumptions on living conditions during the passage of contaminated cloud.

External exposure from ground shine can be assessed from activities of material deposited on the ground using assumptions on living conditions and additional knowledge of environmental transfer processes (e.g. migration into the soil, run-off from paved surfaces) of relevant radionuclides. In addition, ingestion dose can be assessed using knowledge from

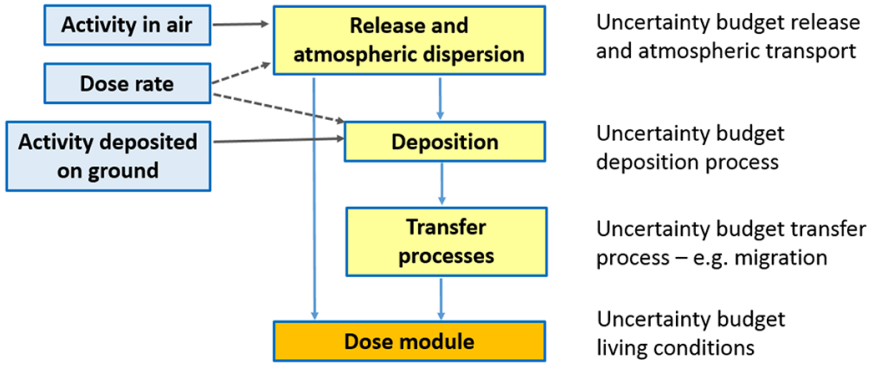

Fig. 1. JRODOS model chain with connected uncertainty budgets and most relevant monitoring data to reduce uncertainties.

radioecological models. However, models for the assessment of ingestion dose use simplified assumptions on consumption of relevant food and drinking water, on agricultural production and on transport and trade routes for the produced food- and feedstuffs. In case of an emergency, ingestion dose will be largely influenced by countermeasures on agricultural production and spontaneous impacts on consumer behaviour.

CONFIDENCE WP1 discussed the main sources of uncertainty in the urgent response phase of an emergency situation and methods to reduce the impact of these uncertainties for decision-making in this phase. Obviously, data from stationary monitoring are helpful to reduce these uncertainties.

During the early response phase, data from stationary monitoring and from mobile monitoring help to reduce uncertainties in dose assessment. This paper focuses on the reduction of uncertainties in the decision-making process in the early response phase using information from environmental monitoring. For prognostic calculations, the focus is on the following endpoints:

- population based dose assessment (from external radiation and from inhalation) and its uncertainty for populated and affected area;

- activities deposited on the ground.

Recommended parameters for monitoring programs in the early phase of an emergency situation are given in Table 3.2 in ICRU Report 92 (2019). Data for ambient dose equivalent rate $(A D E R)$ are very helpful for assessing external exposure and data for radionuclide specific activity concentration are important for assessing exposure due to inhalation. During accidental releases from NPPs, exposure from external radiation and from inhalation is dominated by noble gas and iodine radionuclides, and contributions from other gamma emitters.

In such emergency situations, data from stationary dose rate monitoring give timely and very useful information about the affected area, the level of contamination and the level of exposure to public in the vicinity of probe location. During an accidental release, information from stationary monitoring will reduce uncertainties about the actual release situation and the levels of contamination. Data from stationary air and fallout monitoring will contribute to this information (c.f. Fig. 2).

In such situations, stationary and mobile dose rate monitoring data can be used to assess exposure to the public using dose reconstruction method described in chapter 6.2 of Bleher et al. (2019). 
a)

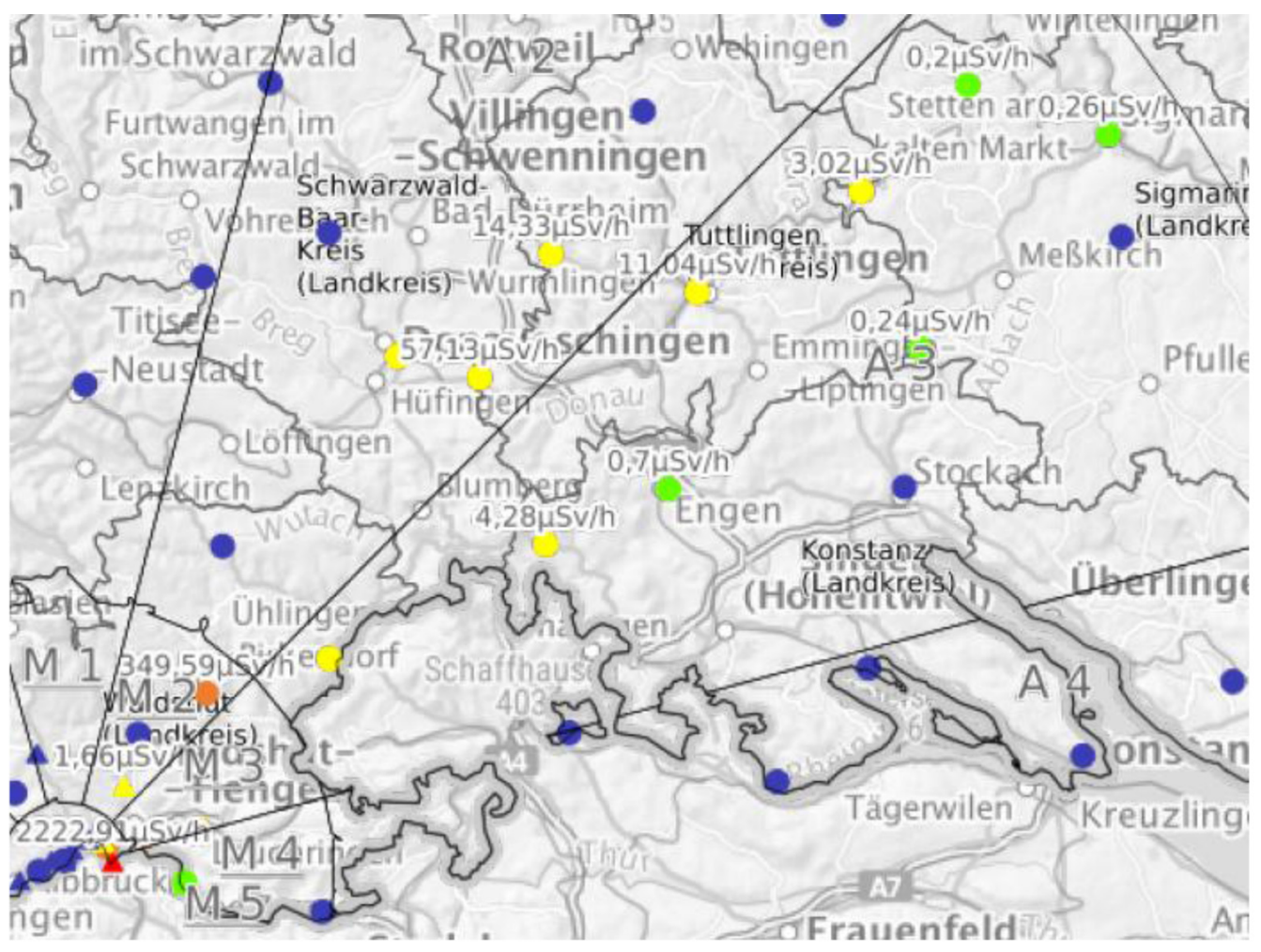

b)

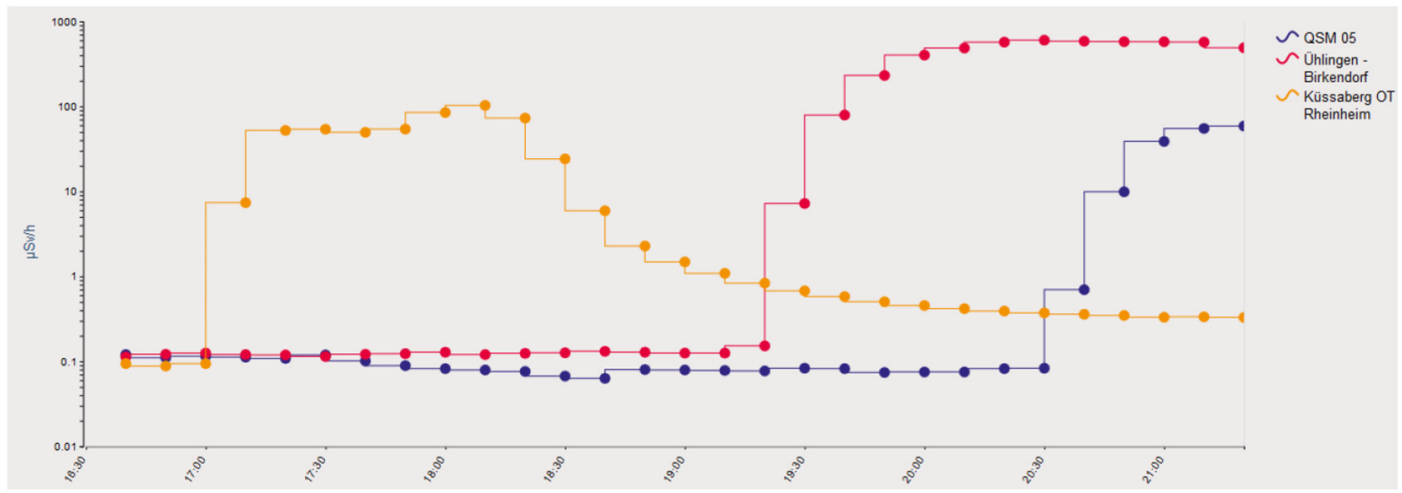

Fig. 2. Simulated data from stationary dose rate monitoring (example from an EP\&R exercise): (a) dose rate in affected area; (b) time evolution of dose rate from selected monitoring stations.

The objectives of this research were:

- identification and quantification of measurement uncertainties of stationary and mobile monitoring systems along with the related challenges;

- identification of the needs of decision support systems to reduce uncertainties in dose assessment using data from monitoring programs;

- development of a generic monitoring strategy for the reduction of uncertainties in environmental monitoring data and more accurate input to the prognostic models in order to optimise assessment of doses to population and environment in early phase.

\section{Stationary monitoring programs}

Most European countries have had installed dose rate monitoring networks for more than 30 years. For member states of the EU, it is obligatory to provide data from these networks via EURDEP data exchange platform.

During an accidental NPP release situation, data from stationary dose rate monitoring give very important information. During the urgent response phase, data from properly designed monitoring networks will indicate the start of a relevant radionuclide release and give information about atmospheric transport and deposition conditions, on affected area and the level of contamination. Uncertainties about actual source term and weather conditions will be reduced.

For this reason, the timely combination of data from national and regional monitoring networks is recommended. Data from spectroscopic dose rate probes in the vicinity would improve the quality of stationary monitoring in case of an emergency situation. Data from such probes will reduce uncertainties about nuclide vector of actual released radionuclides. 
Measurement uncertainties of stationary dose rate monitoring systems are in principal understood. Large efforts are made for characterization of different probes and detectors and discussing harmonization aspects (Dombrowski et al., 2017). In most cases, measurement uncertainties for data from stationary dose rate probes are less than $20 \%$.

However, additional uncertainties due to disturbing objects in vicinity of probes should be taken into account. A MetroERM deliverable discusses classification of different sites with respect to representativeness of measured data (Bleher and Stöhlker, 2017). The proposed site characterisation technique is clearly linked to network harmonisation aspects and to an uncertainty model for ambient dose rate measurements under environmental conditions including disturbing contributions from non-standard probe locations. The proposed technique is adequate for post-release situations, where freshly deposited activity dominates the total dose rate.

Monitoring of airborne radioactivity is carried out routinely and continuously by most European countries. Trace detections of artificial radionuclides are made occasionally with major detection events occurring in 2011 and 2017 with the detections of the radioactivity released from the Fukushima accident, detections of iodine released from what was most likely a radiopharmaceutical production facility and detections of ruthenium from what is conjectured to be related to production of a radionuclide source.

The routine air monitoring programs operate at a time scale that introduces a considerable delay between the passing of a radioactive release and the arrival of the result. The collection time of a sample is usually one week; further delay is caused by the transport and the measurement of the sample. This delay is alleviated by two approaches:

- maintaining the readiness to shorten the sample collection period in an emergency situation;

- installation of on-line monitoring equipment (spectrometers or dose-rate probes) on the sampler to monitor the sample collection.

In an emergency situation, approach 1 will provide accurate knowledge of the air concentration of the released radionuclides present in surface air. It will also lead to a greatly increased volume of samples (even an hourly sample change is prescribed), which will, in turn, place demands on the capacity of the measurement laboratories. This approach will not alleviate the delay of sample transport, which can be substantial when considering the furthest reaches of the monitored area. Important considerations are:

- awareness of the measurement capabilities and capacity in the country/region;

- the capability to deploy a mobile counting laboratory closer to the sampling location.

Approach 2 provides timely early warning of a plume of airborne radioactivity entering the monitored region. The limit of detection is lower than in the spectroscopic monitoring of the environment in conjunction with stationary dose rate monitoring. It is still much higher than the laboratory counting of approach 1. Quantitative analysis of the air concentration of radionuclides is more complicated, and software packages and methods for doing this are not widely available.
Monitoring of fallout is often carried out in conjunction with the monitoring of airborne radioactivity. In emergency situation fallout monitoring may be used to determine the deposition velocity and density of radioactivity from air to ground. The uncertainties arising from environmental factors, characteristics of collectors (especially in the case of passive deposition gauges) and the characteristics of the recipient surfaces are high, especially in the case of dry deposition.

Sources of uncertainties for stationary monitoring methods are investigated in Karhunen et al. (2019) for dose rate, airborne radioactivity and deposition monitoring. The relevance of the uncertainties in an emergency and decisionmaking, especially in the determination whether OIL levels are exceeded, are summarised in Table 1.

\section{Mobile monitoring programs}

During the urgent response phase of an emergency, data from stationary monitoring systems provides information to improve understanding of the situation and to reduce uncertainties for dose and risk assessment. Major release scenarios for radiological emergencies typically lead to large gradients of observed dose rate near the location of the release. In addition, large gradients can be expected in case of precipitation events during the atmospheric dispersion of the contaminated cloud. In both cases, spatial interpolation of measured data leads to large uncertainties. This is also true for calculated data derived from measured data $-e . g$. for assessed exposure for population of affected area using dose reconstruction techniques (see chapter 6.2 of Bleher et al., 2019). To reduce corresponding uncertainties, additional mobile monitoring is needed.

More advanced spatial interpolation methods will not reduce this type of uncertainties significantly. Thus, decision support systems should avoid these models. In most emergency situations, data from mobile monitoring should be used in combination with data from stationary monitoring according to Figure 3. Simple spatial interpolation should be used to analyse the uncertainty budget of dose assessment methods. High uncertainties due to spatial interpolation can be effectively reduced by additional mobile dose rate monitoring data. Thus, optimization of the planning process for additional measurements by mobile teams should include information about the uncertainties due to spatial interpolation.

In the early response phase, the affected area could be derived from the combination of prognostic data (modelling) and stationary monitoring data (routine monitoring). Additional measured data from mobile teams are needed in regions, where spatial interpolation techniques of observed data lead to large uncertainties.

This additional information is relevant especially in populated and severely affected areas. To improve the data basis for dose assessment for the public, mobile teams with the following equipment are recommended:

- air-borne dose rate monitoring teams - e.g. helicopter based aero-gamma systems for long range surveys and drone-based aero-gamma systems for short range surveys; - car-borne dose rate monitoring teams - e.g. plastic scintillator or NaI scintillator; 
Table 1. Uncertainties in monitoring data and methods to reduce uncertainties.

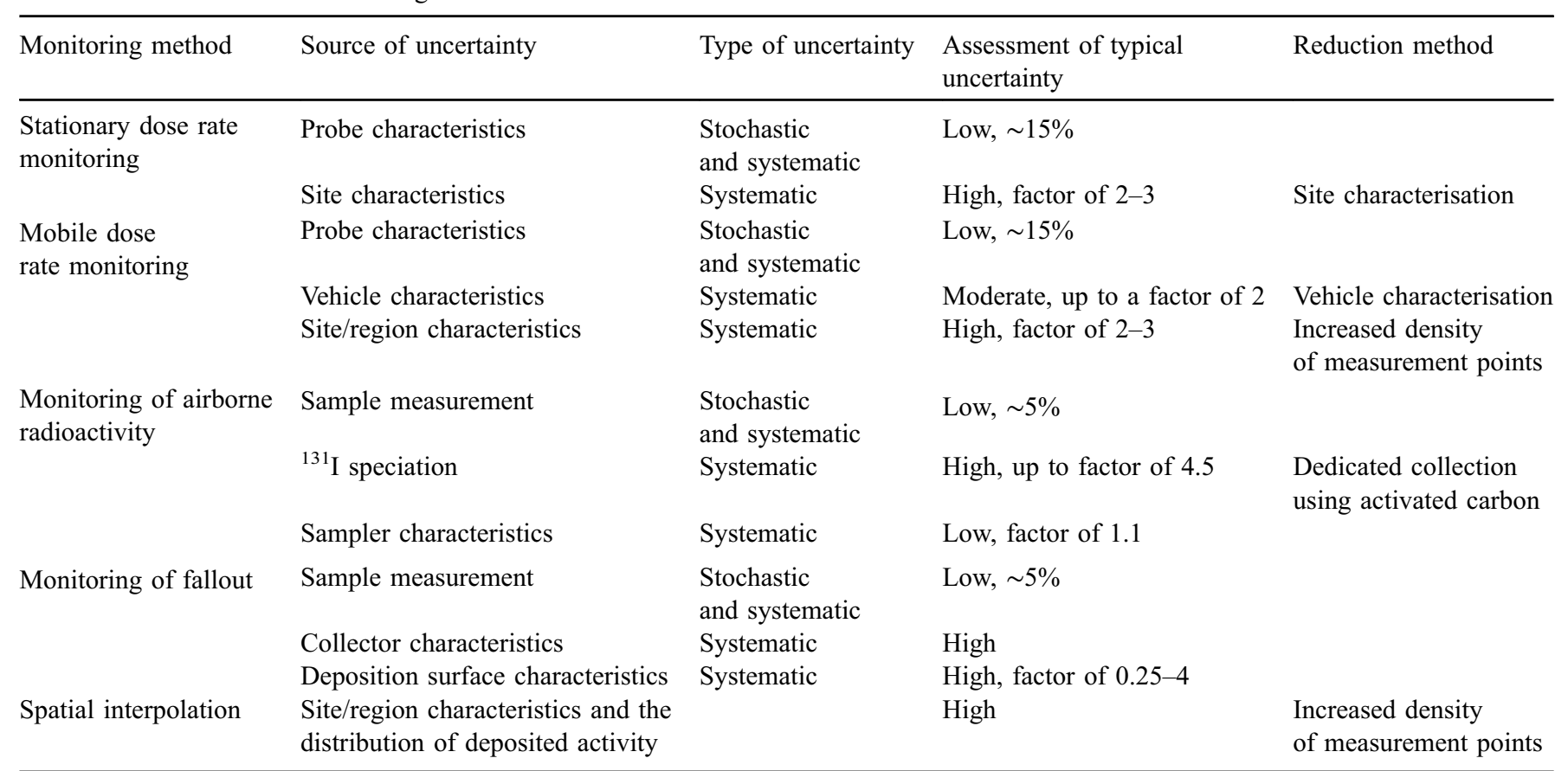

The qualitative assessment is here classified as: high: can decisively sway a decision, e.g. a systematic underestimation would cause a significant exceedance of an OIL level to go un-noticed; moderate: will cause a careful deliberation of a decision; low: no impact on a decision.

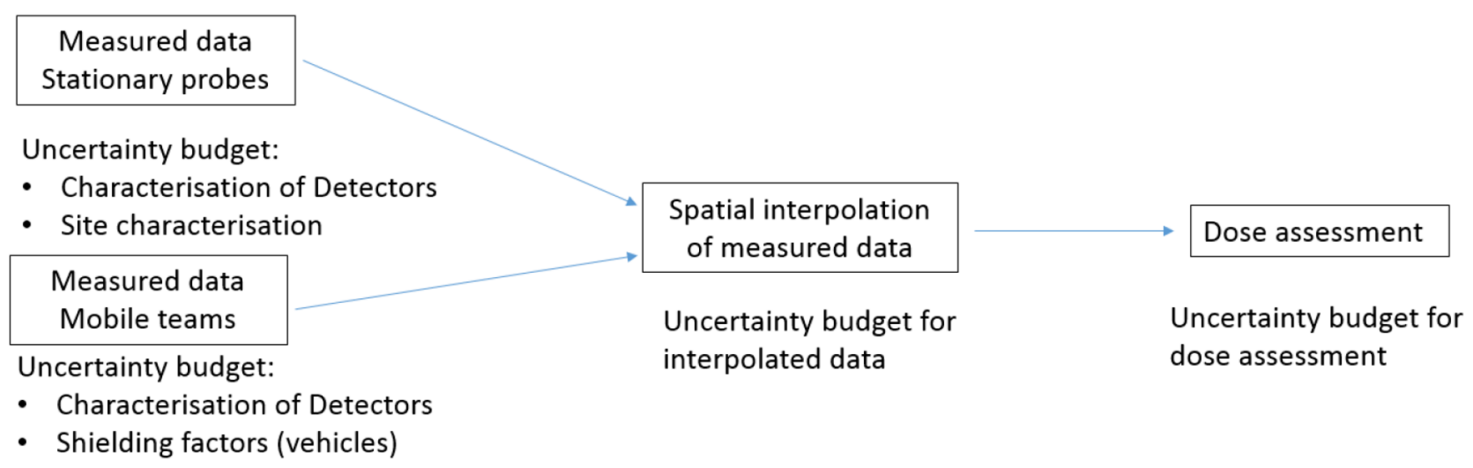

Fig. 3. Uncertainty reduction for dose reconstruction using mobile monitoring and spatial interpolation.

- teams equipped with in situ gamma-spectrometry systems - e.g. HPGe detectors, additional hand-held dose rate probes and mobile aerosol filters.

The monitoring of environmental gamma dose rate within the affected areas will allow the estimation of doses for individuals who were within (or remain within) the affected areas. This monitoring will enable decisions to be made to remove any remaining people from areas with high dose rates and to restrict access to these areas. It will also help to confirm that zone boundaries have been correctly located (or indicate where changes to zone boundaries are needed).

Monitoring of environmental contamination levels within the affected areas will provide information on the spread of environmental contamination and will help in the control of exposure to internal contamination. Contamination monitoring provides another important input to decisions about the placement of zone boundaries (TMT Handbook, 2009). That is why, the accuracy of all measurements and the obtained monitoring data is crucial.

The planning process for additional measurements by mobile teams should reflect radiation protection issues for the teams, the needs of decision-making process and the benefit of additional data for information of the public. For example, it is recommended to deploy mobile autonomous probes for gamma-dose measurements to complement or expand the stationary networks, where possible. This would increase the spatial resolution of monitoring, increase the area coverage of monitoring and to get more precise and accurate data for better dose assessment for population and workers (fire fighters, rescue teams, etc.).

In WP2, monitoring strategies of some European and Scandinavian countries (Germany, Finland, Norway and France) were compared for common aspects. Monitored 


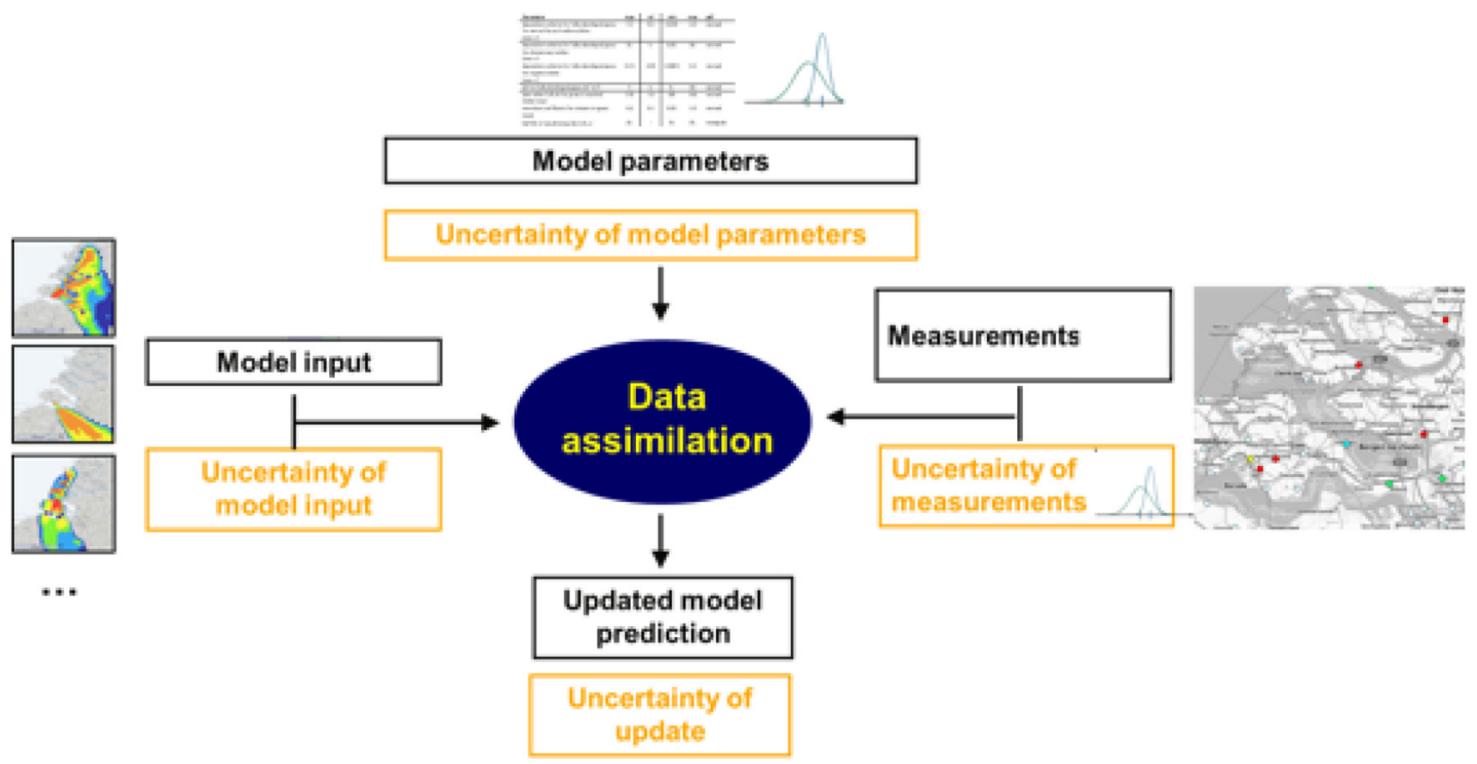

Fig. 4. Reduction of uncertainties in dose assessment using data assimilation techniques to include monitoring data.

quantities and methods of stationary monitoring networks are assessed, including their uncertainties and suitability for use in an emergency situation (Tab. 1). Capabilities of mobile measurements by airborne, carborne and portable systems are considered, including their uncertainties.

Nalbandyan-Schwarz et al. (2019) give an overview of different mobile monitoring systems, their capabilities and applications in emergency response phases as well as discussing challenges related to the measurements' uncertainties. The report also provides suggestions and recommendations for an optimized monitoring strategy that will reduce uncertainties of mobile measurements and get more accurate monitoring data for prognostic models and assessment of doses to population and the environment in the post-release and transition phases.

\section{Reduction of uncertainties in dose assessment using monitoring data}

During the pre-release phase of an emergency situation, decisions on urgent protective actions for the public have to rely on assumptions or predicted information about the potential source term and weather conditions. During the release phase, data from stationary monitoring give additional information about the beginning of the release and on the timescale and the amount of released radionuclides. In addition, the comparison of predicted and observed data may indicate deviations from real conditions for atmospheric transport and deposition process. Monitoring data should be used to check decisions on protective actions. If necessary, updated decisions e.g. for sheltering of the public should be made. For example, modern geographic information systems can be used to overlap maps of predicted dose rate with observed dose rate. In addition, time evolution charts may be used to compare predicted and observed data.

More advanced methods for the combination of model predictions and monitoring data use data assimilation techniques (Fig. 4). Within the JRODOS model chain, data assimilation capabilities can be introduced at several modelling steps. In Bleher et al. (2019), adata assimilation for the Food Chain and Dose Module of JRODOS, FDMT is described. The main focus here is on updating the total deposition on the ground and on all kinds of plant surfaces based on measurements of gamma dose rates. Furthermore, radionuclide concentrations in different feed- and foodstuffs - as modelled with FDMT - could be updated based on direct measurements of these quantities. It contains a description of the underlying deposition model of JRODOS, then the model uncertainties and the formulation of the deposition model in the so-called state space are shown. Furthermore, the Ensemble Kalman filter (Kalman, 1960) and its initialisation within the deposition model are briefly described. Finally, an example for data assimilation with the Ensemble Kalman filter within the deposition model is presented. This example demonstrates how data assimilation approaches can be used for reducing the uncertainty of model predictions making best use of monitoring data.

Furthermore, a dose reconstruction tool was developed in recent years. Using environmental monitoring data, the tool DosRek provides the basis for a population-based dose assessment. It can therefore be used to meet the aims of WP2.1 in order:

- to generate ground contamination maps from radiological monitoring data;

- to assess individual dose histories based on all available radiological monitoring data.

In addition, the aim of this tool is to ensure that the EU standards regarding the assessment of doses and thus the determination of the effectiveness of implemented measures and the comparison of doses with existing reference levels are met. The DosRek tool was developed as a module of the decision support system JRODOS and can be used within JRODOS to identify critically exposed groups or individuals 
for subsequent medical surveillance, long-term health monitoring or epidemiological studies. A detailed documentation of the modelling concept and the fields of application of the DosRek tool is available in German. A short description of the main features of the tool and the German dose assessment approach is summarized in Bleher et al. (2019).

\section{Citizen engagement in dosimetry- evaluation of apps for dose-rate measurements using the camera sensor of the smartphone}

The increasing commercial availability of low-cost and easy-to-use radiation detectors has created new opportunities for citizens to be actively engaged in radiation protection and environmental monitoring by making their own radiation measurements, either on a self-organized basis or guided by experts. This has led to crowd sourcing and citizen science approaches being imported into the field of radiation protection, where crowdsourcing is to be understood as a process where voluntary contributions from a large group of unknown individuals ("the crowd") are collected and used for a specific purpose, for instance the creation of collective data maps by sharing geo-localization and measurements.

In principle, citizens have a choice of two types of technologies for radiation measurements. Firstly, there are external detectors available, e.g. based on Geiger-Müller counters, that can be coupled to a smartphone with a dedicated smartphone application. And secondly, smartphone applications can be used that turn the smartphone itself into a radiation detector without any additional hardware. This is possible by using the Complementary Metal-Oxide Semiconductor (CMOS) sensor of the in-built camera as a radiation detector, after covering the lens with black tape. The latter technology is less sensitive than dedicated external detectors but is also at the same time much more affordable (a few euros compared to 50500 \&z.euro;). For both detector types, the measurements can be coupled to existing citizen science networks with an online platform to collect and visualize radiation measurements by citizens (e.g. Safecast and OpenRadiation).

In the context of monitoring and dose assessment, described in the previous sections, citizen measurements can potentially be of dual use. By providing dose-rate maps through crowdsourcing and online platforms, they can complement the existing stationary and mobile monitoring data, with an expected focus on densely populated areas. And since the dose rate measurements can be continuously integrated and the device is usually held close to or on the body during measurement, an individual dose assessment is possible, complementing the results of the dose reconstruction tool and/or the techniques of individual dose measurement, as described e.g. in López et al. (2020). A prerequisite for this is, however, that the performance and quality of such non-expert measurements can be assured or evaluated.

As a contribution towards this goal, the characterization of two applications for dose-rate measurements with the smartphone CMOS sensor was carried out within CONFIDENCE. The working principle behind the apps is that, after blocking out any visible light by covering the camera with black adhesive tape, a measurement can be run through a video record where every interaction of radiation (photons) with the chip is observed as an intense signal in a certain pixel (bright spot). The dedicated software then analyses each video frame by counting the number of spots, at a frame rate depending on the smartphone model. The sum of detected particles in selected time periods is given as a number of counts, and when available, conversion from counts to dose-rates is then performed through appropriate calibration factors. Two applications, "GammaPix" and "RadioactivityCounter", were investigated, installed on 14 different mobile phones of the most common brands sold in 2017 and covering the low, medium and high price range. Performance parameters studied were the dose-rate response for dose-rates between 2 to $1000 \mu \mathrm{Gy} / \mathrm{h}$, the background assessment and the energy and angular dependence of the camera detector. Since "GammaPix" lacked a proper calibration for all phone models tested, resulting in underestimating the nominal reference dose-rate values by at least an order of magnitude, more focus was put into evaluating the "RadioactivityCounter" app. It was found that performance of the app (background radiation level and the dose-rate sensitivity) was dependent on the noise value " $n$ " of the sensor, and that phones could be grouped into two groups with $n$ being larger or lower than 10. Devices equipped with cameras of good quality showed low noise levels $(\mathrm{n}<10)$, agreement between nominal and reference dose rate down to $10-20 \mu \mathrm{Gy} / \mathrm{h}$, but larger uncertainties in the data for lower dose rates. Models characterized by higher noise $(n>10)$ resulted to be blind below the threshold of $50 \mu \mathrm{Gy} / \mathrm{h}$. The energy response was investigated in the energy range from $24 \mathrm{keV}$ (mean energy) to $662 \mathrm{keV}$ (Cs-137). As expected for a detector made of a semiconductor with metal components, a strong over-response of up to a factor of 18 for a photon energy of around $60 \mathrm{keV}$ was observed on all the different phones, a property that is consistent with previous studies (Van Hoey et al., 2016). Since for ground contamination it is expected that gamma rays will hit the detector at various angles of incidence, the angular dependence of the sensor was assessed for four phone models. An overall variation of the measured signal at maximum about $10 \%$ for angles of incidence up to $60^{\circ}$ was seen. A full description and discussion of all results can be found in Mafodda and Woda (2020, submitted).

\section{Discussion and conclusions}

Monitoring strategies are designed to answer the information needs for decision-making in a nuclear or radiological emergency. The effective implementation of a monitoring strategy for a given scenario requires that the methods and equipment are sufficiently characterized along with their uncertainties. Besides uncertainties mentioned above, there are still more factors to consider regarding the environmental monitoring methods, e.g. the delays caused by sample transport and counting or the types of radioactivity that can be detected with given methods. Another important aspect is the adequate training and expertise of a mobile team.

Measurement data from environmental monitoring can be used to reduce the uncertainties of dose assessment in the early phase. The key idea is the combination of the monitoring data with prognostic models in a decision support/emergency 
management system. The necessary techniques have to be implemented, incorporated into a measurement strategy and practiced in exercises.

In WP2 measurement uncertainties of stationary and mobile monitoring systems were assessed, including the uncertainty due to iodine speciation in the monitoring of airborne radioactivity. Generally, the uncertainty budget was dominated by the detector environment (sites, vehicles) and by the uncertainty in interpolation but not by the measurement/ detector uncertainty itself. Monitoring strategies were further developed to reduce the interpolation uncertainty by increasing the network density through deployment of mobile measurement systems. Regarding citizen engagement in monitoring and dosimetry, reliable measurements at natural environmental radioactivity or slightly elevated level turned out to be difficult to assess when using the CMOS camera sensor of the smartphone as a detector. However, the smartphones apps are potentially useful to identify regions of high(er) radioactive contamination.

It is suggested that the necessary monitoring networks have to be properly designed and characterized, with quality assurance methods in place, to guarantee the quality of the monitoring data. Mobile monitoring teams should be used to complement the stationary monitoring networks and models in areas where uncertainty of dose to population is high.

Acknowledgements. The work described in this paper was conducted within the CONFIDENCE project, which was part of the CONCERT project. This project has received funding from the Euratom research and training programme 20142018 under grant agreement No. 662287.

Disclaimer (Art. $29.5 \mathrm{GA}$ ). This publication reflects only the authors' views. Responsibility for the information and views expressed therein lies entirely with the authors. The European Commission is not responsible for any use that may be made of the information it contains.

\section{References}

Bleher M, Gering F, Meisenberg K, Stöhlker U, Strobl C, Karhunen T, Nalbandyan-Schwarz A, Jaworska A, Tschiersch J, Manach E. 2019. Report on uncertainty reduction in exposure assessment based on environmental monitoring data, including concept for identifying critically exposed groups. CONCERT Deliverable D9.7. Available from: https://www.concert-h2020.eu/en/Publications.

Bleher M, Stöhlker U. 2017. Site characterisation methods and corrective procedures developed. MetroERM Deliverable D1.5.4.

Dombrowski H, Bleher M, De Cort M, Dabrowski R, Neumaier S, Stöhlker U. 2017. Recommendations to harmonize European early warning dosimetry network systems. JINST 12: P12024.
Ehrhardt J, Weis A. (Eds.). 2000. RODOS: decision support system for off-site nuclear emergency management in Europe. Final report of the RODOS project. Brussels: European Commission. Report EUR 19144, ISBN No. 92-828-9773-7, einschl. 2 CDs mit allen technischen Dokumenten des RODOS Systems.

IAEA. 2018. Arrangements for the termination of a nuclear or radiological emergency. IAEA Safety Standards Series No. GSG11. Vienna: IAEA.

ICRU Report 92. 2019. Radiation monitoring for protection of the public after major releases of radionuclides to the environment. Available from: https://icru.org/content/reports/icru-report-92-ra diation-monitoring-for-protection-of-the-public-after-majorreleases-of-radionuclides-to-the-environment.

Ievdin I, Trybushnyi D, Zheleznyak M, Raskob W. 2010. RODOS reengineering: aims and implementation details. Radioprotection 45 (5 Suppl.): S181-S189.

Gering F, Raskob W, Charnock T. 2010. New model for agricultural countermeasures in RODOS and ARGOS. Radioprotection 45(5): S63-S76. https://doi.org/10.1051/radiopro/2010035.

Kalman RE. 1960. A new approach to linear filtering and prediction problems. J. Basic Eng. 82: 35-45.

Karhunen T, Bleher M, Tschiersch J, Nalbandyan-Schwarz A. 2019. Report on uncertainty reduction in exposure assessment based on environmental monitoring data, including concept for identifying critically exposed groups, D9.7.1 Stationary monitoring systems and their uncertainties. CONCERT Deliverable D9.7. Available from: https://www.concert-h2020.eu/en/Publications.

López MA, Berkovskyy V, Ratia G, Challeton-de-Vathaire C, Davesne E, Eakins J, Franck D, Giussani A, Gregoratto D, Hernandez C, Kulka U, Mafodda A, Marsh JW, Navarro JF, Oestreicher U, PérezB, Sierra I, Woda C. 2020. Improvement in individual dose measurements techniques following nuclear emergencies. Radioprotection 55(HS1). https://doi.org/10.1051/radiopro/2020017.

Mafodda A, Woda C. 2020. External dose-rate measurements based on smartphone CMOS sensors. Radiat. Meas. (submitted).

Nalbandyan-Schwarz A, Karhunen T, Bleher M. 2019. Report on uncertainty reduction in exposure assessment based on environmental monitoring data, including concept for identifying critically exposed groups, D9.7.2 Mobile monitoring systems and their uncertainties. CONCERT Deliverable D9.7. Available from: https://www.concert-h2020.eu/en/Publications.

Raskob W, Gering F. 2010. Key improvements in the simulation modelling for decision support systems developed in the EURANOS project. Radioprotection 45(5): 149-159.

TMT Handbook. 2009. Triage, monitoring and treatment of people exposed to ionising radiation following a malevolent act. ISBN 978-82-90362-27-5, pp. 556. Norway.

Van Hoey O, Salavrakos A, Marques A, Nagao A, Willems R, Vanhavere F, Cauwels V, Nascimento LF. 2016. Radiation dosimetry properties of smartphone CMOS sensors. Radiat. Prot. Dosim. 168: 314-321.

Cite this article as: Bleher M, Gering F, Stöhlker U, Karhunen T, Nalbandyan-Schwarz A, Woda C, Mafodda A. 2020. Reduction of uncertainties in exposure assessment based on environmental monitoring data. Radioprotection 55(HS1): S81-S88 